\title{
Database Programming Technology Based on Computer Software Engineering
}

\author{
Peichun Suo \\ Yunnan College of Business Management, 650304, China
}

Keywords: Computer Software Engineering, Database, Programming Technology

\begin{abstract}
Computer software engineering has become one of the most popular industries in society, and its role in social development is obvious to all. From the point of view of the current computer software engineering industry, although the development process is accelerating and new achievements are emerging, there are still many problems, which are very unfavorable to the better and faster development of computer software engineering. In these problems, the most concerned is the application of database programming technology, because of the existence of technical problems, the database cannot play its due value. This thesis focuses on database programming based on computer software engineering.
\end{abstract}

\section{Introduction}

Database programming in computer software development Is a new technology, can provide the computer software engineering industry development important Resources available.However, in the application of database technology, various kinds of Factors affecting the technology lead to specific Application There will be Many The problem Appear.To ensure the application value of database technology, we must take effective measures to solve the problems we are facing.Programmer We should attach great importance to this and take it Work As a priority.This requires programmers to improve their cognitive ability, to upgrade and innovate database technology, and to promote the better development of computer software engineering. (Figure 1: Computer Database Management page)

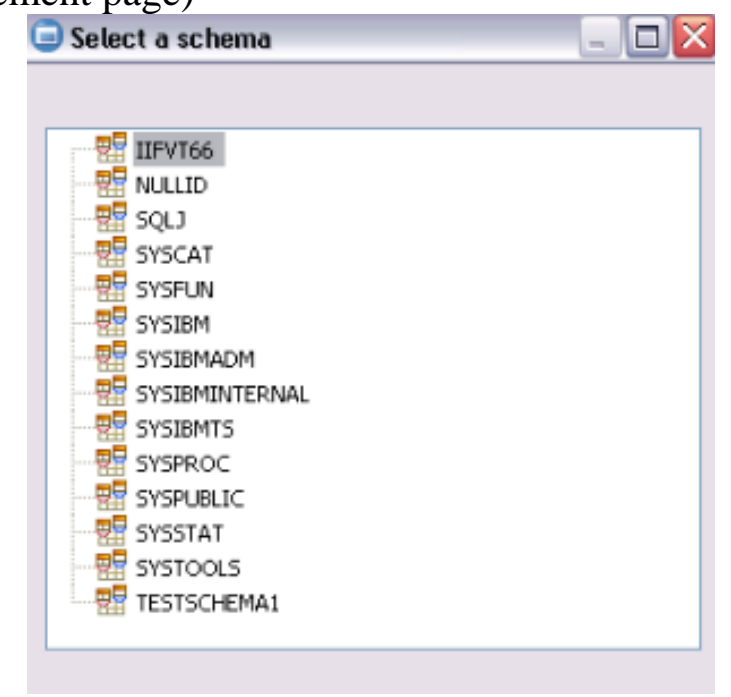

Figure 1 Computer database management page

\section{The Role of Programming Techniques in Computer Databases}

\subsection{Programming Techniques in Computer Databases for Authentication}

At present, in computer software engineering, database technology is widely used, which plays a role in promoting the rapid and stable development of computer software engineering programming industry. Programming technology can be used for identification, which is to authenticate the access.From Procedures From its own point of view, it is Open Sex. All users can access, and the 
forms of access are varied. To ensure the security of access users and information, the identity of users needs to be verified[1].

The implementation of this technology is mainly reflected in the authentication used by computer access, but also to verify the identity of port computer access. However, in the specific implementation, even if the authentication method is different, the user does an operation, that is, through the link established by HTTP, the wide password input into the way to use the account login. However, if you check the user's account number and password and then compare the information in the database, if the two do not want to match, the method will be banned. If matched, it automatically enters the access space. In the user operation, the password can be saved in the server's private file to prevent the password from being stolen. The application of programming technology in the computer database can protect the user information and ensure the safe use of the database, especially the enterprise users, can effectively prevent the loss of internal information or malicious tampering of the enterprise, avoid the resulting economic losses, the management of the enterprise is more convenient, and the economic benefits will also be improved. (Figure 2: Computer Database User Authentication)

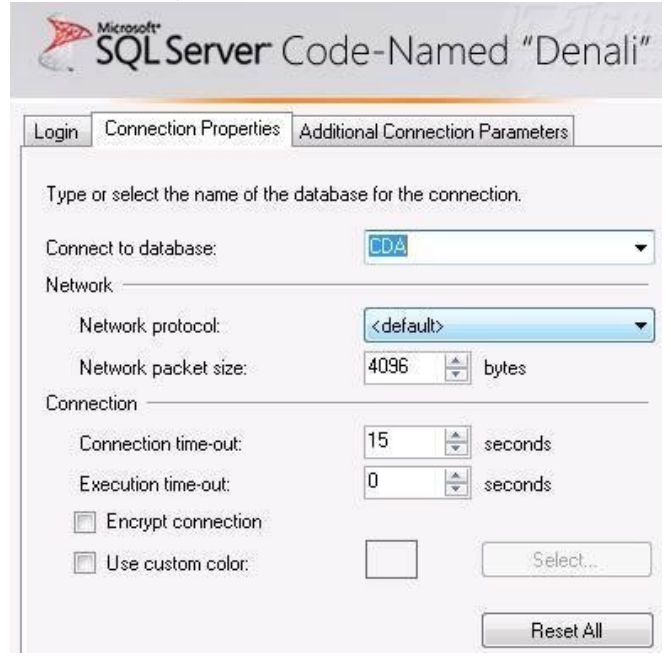

Figure 2 Computer database user authentication

\subsection{Programming in Computer Databases Can be Used to Hide Information}

In the application of programming technology, we can also play the role of hiding some information. When connecting network communication lines, under the influence of network address conversion technology, the URLs connected by users in the network will be uniformly hidden and displayed as public address access, which is the role of programming technology in the concealment. In enterprise network management, this function plays an important role. Using this technology, the enterprise manager can directly access the network platform of the enterprise functional department through the computer network. However, if the external computer network With Internal enterprise network Connection, operation will be contained, this can be Make the enterprise Internal information Higher confidentiality, avoid The problem of information disclosure caused Level of enterprise information security Some improvement.

\section{Methods of Establishing Database Files in Computer Software Engineering}

\subsection{Computer Software Engineering Basic Conditions for Establishing Database Files}

During the process of establishing database files in computer software engineering, it is necessary to check Related Information to ensure that information is available Normal reading In order to consider the establishment of database files, this is the establishment of database files Required basic condition, otherwise this operation cannot be performed. If found Data information No way Normal reading, even if established Database files, too Failure, so, programmers need to recognize Between the two Is the condition and the result Relationships[2]. In addition, when 
establishing database files, programmers need to compare the information in the database, comprehensive analysis, detailed understanding, but also carefully observe some of the problems found in the reading process, combined with the problems found in the selection of the corresponding database establishment, so that the technical problems are effectively resolved, database file establishment is more convenient.

\subsection{Computer Software Engineering Method of Establishing a Database}

Computer software engineering A scientific and effective approach is needed to establish the database. Once you have identified the type of database file, you can create the database. However, the method of creating a database is not immutable and can be chosen flexibly as needed. When the database file creation is completed, it also needs to be optimized and improved as needed. The improvement work is mainly due to the database itself has functional defects, cannot meet the requirements, or some computers in the process of running problems. Technically Improved database More powerful, the operation of the computer Quality and Speed Have been improved, database files can Normal use to prevent Computer Application Database usage exists Question.

\section{Database Programming Technology Used in Computer Software Engineering}

\subsection{Database Programming Technology Design and Development}

In order to make full use of the database programming technology in computer software engineering, it is necessary to design the programming technology and develop the corresponding software. When a database is applied, a number of problems are caused by various factors. To solve these problems, we should go back to the programming stage and reprogram the database, so that the problems in database application can be solved effectively. However, if you go back to the programming stage, it takes a lot of time and effort to rewrite the program, so the programmer should solve the problem of the early stage of programming, and make effective data storage system, which can avoid the problem of secondary modification.[3].

In the process of programming, programmers should choose the corresponding programming technology according to different software, and the characteristics of different software should be fully taken into account in the specific operation. In addition, for some problems that need to be agreed in the process of programming, we need all the programmers involved in the program to discuss together, to analyze the existing problems in detail, to make a summary of the key points, to solve the problems effectively under the premise of ensuring the smooth operation of the software, to avoid the various problems in the process of software operation, so that the programming technology can play a better role.

\subsection{Database Documentation Encryption Technology}

Using encryption technology in database programming, you can encrypt files and maintain the security of database files. Although the network is open, but chat records, search information and browsing history is still involved in privacy issues, security, need to do a good job of encryption. As a place to store this information, it is necessary to use encryption technology to keep secret.Once the confidentiality of the database Not very good, will make the user's Privacy information stolen, or even tampered with without the user, resulting in threats to the safety of the user.Application of programming technology, can Play its encryption function, make Database security performance Article, User Personal Information Increased security can effectively avoid privacy Leaked.The use of programming technology to encrypt database information is mainly reflected in two aspects: first, programmers can use programming technology to organize all kinds of information in the database to organize, and analysis, encryption processing, for basic information and privacy information storage should be strictly separated. Encrypting more privacy information in the database can protect the information very well. If Privacy Information Disclosure The problem can be alerted to the programmer as soon as possible and in time Effective measures To avoid large-scale disclosure of information[4]. The second is encryption to the database, the database needs to implement 
encryption protection, but also need to implement multi-layer encryption protection, so that the database information leakage problem is eliminated. For example, programmers can divide encryption into three levels: the first level is the unified execution of system authentication; the second level is the way of using computer access authentication; the third level is the user's own authentication, which can require the user to design his own login password to match his own account. When logging in, system programming can verify the matching of passwords and accounts, making database information more secure. However, for encryption protection to adopt a multilevel design, programmers must pay attention to the order between the encryption layers, avoid the contradiction between the encryption layers, and constantly optimize the encryption layer.(Figure 3: Computer Database Access Interface)

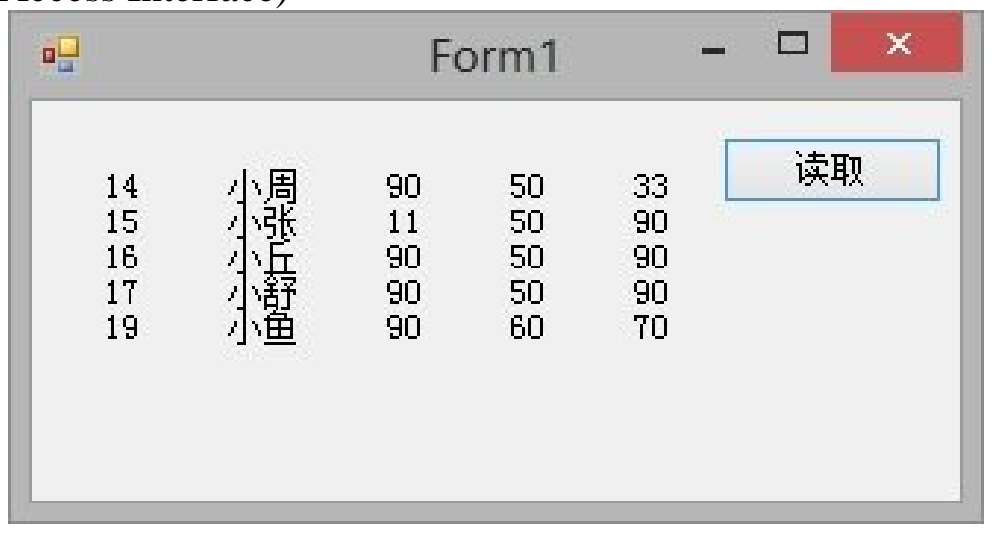

Figure 3 Computer database access interface

\section{Conclusion}

Through the above research, it is clear that the application of programming technology in the computer database should be reasonably applied according to the specific needs. In the concrete implementation, it is necessary to fully understand all the application value of the disassembly technology, including the content of the technology application and the corresponding table, from the content to the application form.Focus on analysis Computer software engineering programming technology, but also from the actual needs to go deep Explore, will Editing technology Application of Strategy proposed for relevant staff For reference.

\section{References}

[1] Gu, Jiayin., Wu, Min. Practice and Exploration of Underground Pipeline Construction Technology in Municipal Construction. Juye, no. 07, pp. 366-366, 2019.

[2] Xu, Lijin. Research on Innovation and Improvement of Computer Software Engineering Management. Computer Products and Circulation, no. 08, pp. 168-168, 2017.

[3] Zhang, Jingbo. Computer Database Backup and Recovery Technology. Electronic Technology and Software Engineering, no. 15, pp. 171-171, 2017.

[4] Sun, Tianbin. Selection of Programming Language in Computer Application Software Development. Electronic Technology and Software Engineering, no. 03, pp. 60-61, 2017. 